\title{
Dépôt par ablation laser UV nanoseconde pour la réalisation de composants Télécom
}

\author{
C. Champeaux, T. Delage, J.C. Orlianges, A. Catherinot, A. Pothier ${ }^{1}$, J.F. Seaux ${ }^{1}$, \\ P. Blondy ${ }^{1}$, V. Madrangeas ${ }^{1}$ et D. Cros $^{1}$
}

\author{
Centre de Projet " Films Minces et Microdispositifs pour Télécommunications », \\ Sciences des Procédés Céramiques et de Traitements de Surface, SPCTS, \\ UMR 6638 du CNRS, Faculté des Sciences, 123 avenue Albert Thomas, \\ 87060 Limoges cedex, France \\ ${ }^{1}$ Centre de Projet « Films Minces et Microdispositifs pour Télécommunications », \\ Institut de Recherches sur les Communications Optiques et Microondes, IRCOM, \\ UMR 6615 du CNRS, 123 avenue Albert Thomas, 87060 Limoges cedex. France
}

\begin{abstract}
Résumé : Devant la multiplication des standards et des normes de systèmes de télécommunications sans fil, il apparaît un besoin important de composants accordables. Diverses solutions peuvent être envisagées parmi lesquelles, l'introduction de nouveaux matériaux ct/ou de nouvelles structures, Micro-systèmes Electro-Mécaniques (MEMS-RF), capables de modifier les propriétés électriques des composants qui en sont constitués. Toutes les applications visées font appel à des techniques de conception adaptées, nécessitant la réalisation de films minces, aux propriétés électriques et mécaniques parfaitement contrôlées. C'est pourquoi, le procédé de dépôt par ablation laser est un bon candidat notamment car il peut s'intégrer dans les chaînes de fabrication micro-électronique. L'étude que nous développons concerne la réalisation de composants passifs hyperfréquences accordables en utilisant des techniques basées, sur l'élaboration par ablation laser UV nanoseconde ( $\mathrm{KrF} 248$ $\mathrm{nm}$ ), d'une part, d'hétérostructures (bi et tri-couches) de matériaux ferroélectrique $\mathrm{Ba}_{2 / 3} \mathrm{Sr}_{1 / 3} \mathrm{TiO}_{3}$ et supraconducteur $\mathrm{YBa}_{2} \mathrm{Cu}_{3} \mathrm{O}_{7-\delta}$ et d'autre part, de films pour la fabrication de MEMS.
\end{abstract}

\section{INTRODUCTION}

Les systèmes de communications sans fil, actuellement en plein essor, ont su trouver de nombreuses applications dans la vie quotidienne, comme dans la téléphonie mobile, la distribution multimédia par faisceau hertzien ou encore les réseaux locaux informatiques. Les fournisseurs de ces équipements doivent faire face d'une part à l'arrivée de nouveaux standards, de nouvelles fréquences d'utilisation, et d'autre part à des objectifs de coût du produit final réduit. Ceci entraîne pour les composants utilisés une exigence sans cesse croissante en terme de performances et de degré d'intégration. Il est donc nécessaire d'imaginer une électronique analogique configurable selon le dispositif dans lequel elle est utilisée. Aujourd'hui, plusieurs possibilités sont envisageables, parmi lesquelles, l'introduction de nouveaux matériaux et de nouvelles structures capables de modifier les propriétés électriques des composants qui en sont constitués

L'étude que nous développons concerne la réalisation de composants passifs $R F$ et hyperfréquences accordables, en utilisant des techniques basées sur l'introduction de matériaux aux caractéristiques variables, les ferroélectriques, et des procédés de fabrication originaux de nouveaux composants. Ces nouvelles structures, Micro-systèmes Electro-Mécaniques (MEMs-RF), sont des systèmes agiles intégrant des éléments mécaniques déformables, fabriquées en utilisant les technologies micro-électroniques.

Chaque application nécessite la réalisation de films minces complexes, aux propriétés électriques et mécaniques parfaitement contrôlées. Parmi les différents procédés de réalisation de couches minces, la technique de dépôt par ablation laser (ou PLD), célèbre depuis son succès dans l'élaboration de films supraconducteurs d'excellentes qualités, est un bon candidat, qui, de plus, peut sintégrer dans les procédés de fabrication de micro-électronique. 


\section{INTRODUCTION DE NOUVEAUX MATERIAUX - RÉALISATION D'HÉTÉROSTRUCTURES}

La réalisation de composants hyperfréquences accordables fonctionnant à basse température nécessite l'utilisation de matériaux supraconducteurs [1]. Mais, du fait même du fonctionnement à basse température, une intervention directe sur le composant, par exemple pour un réglage en fréquence, est difficile à mettre en cuvre par des techniques classiques. C'est pourquoi, l'introduction de matériau ferroélectrique permet d'envisager d'effectuer l'ajustement par une modification de la tension continue appliquée à certains éléments du système. Le titanate de baryum et strontium $\mathrm{Ba}_{\mathrm{x}} \mathrm{Sr}_{1-\mathrm{x}} \mathrm{TiO}_{3}$ (BST) est l'un des matériaux ferroélectriques les plus utilisés dans la conception de dispositifs hyperfréquences accordables en fréquence [2]-[3].

\subsection{Couches minces ferroélectriques $(\mathrm{Ba}, \mathrm{Sr}) \mathrm{TiO}_{3}$}

Nous nous intéressons à la réalisation de couches minces ferroélectriques de $\mathrm{Ba}_{\mathrm{x}} \mathrm{Sr}_{1-\mathrm{x}} \mathrm{TiO}_{3}$ avec $\mathrm{x}=2 / 3$. De la valeur du taux de substitution $x$ dépendent la permittivité et la température de Curie [4]. Les films sont réalisés dans une enceinte ultravide, par ablation laser de cibles frittées. Le faisceau d'un laser à excimères $\mathrm{KrF}$, émettant à $248 \mathrm{~nm}$ avec une durée d'impulsion de $15 \mathrm{~ns}$ et une fréquence de $10 \mathrm{~Hz}$, irradie la cible avec une fluence de l'ordre de $3 \mathrm{~J} / \mathrm{cm}^{2}$. Les espèces éjectées perpendiculairement à la surface de la cible sont collectées sur le substrat chauffé placé en regard. Les dépôts sont réalisés sous 0,3 mbar d'oxygène et refroidis sous 330 mbar d'oxygène. L'évolution de la structure cristalline en fonction de la température de dépôt sur substrat $\mathrm{MgO}$ et $\mathrm{SrTiO}_{3}$ a été étudiée dans la gamme $500-800{ }^{\circ} \mathrm{C}$. La phase orientée (100) de $\mathrm{Ba}_{2 / 3} \mathrm{Sr}_{1 / 3} \mathrm{TiO}_{3}$ croît à partir d'environ $600^{\circ} \mathrm{C}$ sur $\mathrm{SrTiO}_{3}$ [5] comme sur $\mathrm{MgO}$ [6].

La caractérisation des échantillons dans le domaine hyperfréquence (à $12,5 \mathrm{GHz}$ ) a nécessité la conception d'un dispositif spécifique (IRCOM). La mesure de la permittivité à température ambiante s'effectue dans une cavité cylindrique. Sur chacune de ses bases est placé un résonateur. L'échantillon à caractériser est placé entre ces deux résonateurs. La mesure de la permittivité diélectrique est issue de la variation de la fréquence de résonance de la cavité avec et sans dépôt [7]. La valeur de la permittivité relative $\varepsilon_{\mathrm{r}}$ atteint 1120 pour les échantillons d'épaisseur $300 \mathrm{~nm}$, réalisés à $800^{\circ} \mathrm{C}$ sur $\mathrm{MgO}$ et est très fortement dépendante de l'orientation cristalline. Il est donc impératif de maîtriser parfaitement les conditions expérimentales afin d'obtenir des dépôts mono-orientés, qui peuvent ensuite être utilisés pour la réalisation de composants. En effet, l'un des objectifs est ici la réalisation de composants hyperfréquences à base de supraconducteurs fonctionnant à basse température. C'est pourquoi nous nous intéressons à la réalisation de multicouches de matériaux supraconducteur $\mathrm{YBaCuO}$ et ferroélectrique BST.

\subsection{Multicouches supraconducteur $\mathrm{YBC}$ / ferroélectrique $\mathrm{BaSrTiO}_{3}$ (BST)}

Des bi-couches $\mathrm{YBa}_{2} \mathrm{Cu}_{3} \mathrm{O}_{7-\delta} / \mathrm{Ba}_{2 / 3} \mathrm{Sr}_{1 / 3} \mathrm{TiO}_{3}$ et $\mathrm{Ba}_{2 / 3} \mathrm{Sr}_{1 / 3} \mathrm{TiO}_{3} / \mathrm{YBa}_{2} \mathrm{Cu}_{3} \mathrm{O}_{7-\delta}$ sont réalisées sur substrat monocristallin $\mathrm{MgO}$. Chaque couche, d'épaisseur $300 \mathrm{~nm}$ est réalisée dans des conditions similaires (env. $800{ }^{\circ} \mathrm{C}, \quad 0,3$ mbar d'oxygène, 3

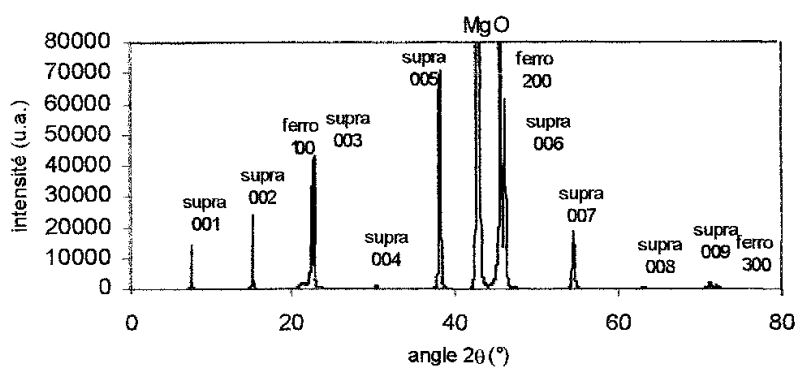

Figure 1: Spectre de diffraction de rayons $\mathrm{X}$ de bi-couche $\mathrm{BST} / \mathrm{YBC} / \mathrm{MgO}$ (ou YBC/BST/MgO) $\mathrm{J} / \mathrm{cm}^{2}$ ). Diverses expériences ont été menées afin de définir les conditions optimales d'élaboration de bicouches épitaxiées. Il s'avère que toutes les bi-couches YBC/BST et $B S T / Y B C$ réalisées en deux étapes, c'est-à-dire que le second dépôt n'est effectué qu'après le refroidissement complet, sous quelques centaines de mbar d'oxygène du premier dépôt, sont épitaxiées, orientées (001) comme en témoigne le diagramme de diffraction de rayons $\mathrm{X}$ de la figure 1. Les largeurs à mi-hauteur des pics (005) d'YBaCuO et (200) de BST inférieures à $0,2^{\circ}$ attestent de la qualité cristalline de chaque 
couche. Afin de tester en le fonctionnement dans le domaine hyperfréquence de ces hétérostructures, la réalisation d'un composant est nécessaire.

\subsection{Capacité supraconducteur / ferroélectrique BST / supraconducteur}

Le premier composant réalisé est une capacité ferroélectrique entre électrodes supraconductrices. Ses dimensions sont issues de simulations, afin de répondre aux exigences des expériences hyperfréquences. II a été réalisé intégralement in situ. Le tri-couche est parfaitement épitaxié (cf figures 2 et 3 ).

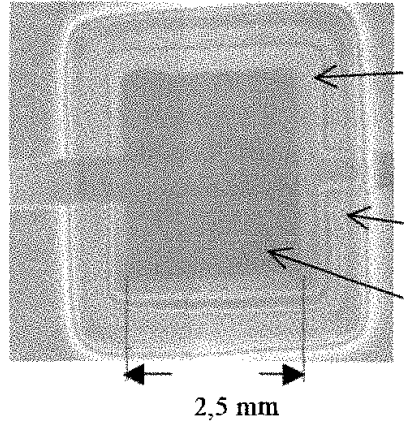

Figure 2: Photographie d'une capacité

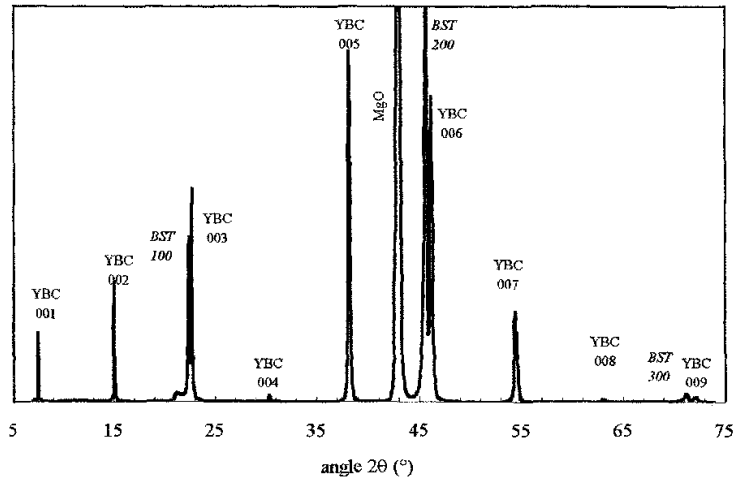

Figure 3: Diffractogramme $\mathrm{X}$ de la capacité

Les paramètres $\mathrm{S}$ de la capacité ont été mesurés à $77 \mathrm{~K}$ pour différentes tensions appliquées entre les deux électrodes. La variation de la permittivité du ferroélectrique induit une variation de la fréquence de résonance, aux environs de $15 \mathrm{GHz}$ (cf figure 4). En supposant que la fréquence de résonance est uniquement d'origine capacitive $\left(f=\frac{1}{2 \pi \sqrt{L C}}\right)$, on peut écrire alors $f=\frac{A}{\sqrt{\varepsilon_{r}}}$ où A est une constante et $\varepsilon_{\mathrm{r}}$ la permittivité relative, et déterminer le rapport des permittivités $\varepsilon_{\mathrm{r}}(\mathrm{V}) / \varepsilon_{\mathrm{r}}(0)$ aux tensions $\mathrm{V}$ et 0 (fig.5). Il s'ensuit qu'un champ appliqué de $1 \mathrm{~V} / \mu \mathrm{m}$ induit une variation relative de permittivité de l'ordre de $0,1 \%$.

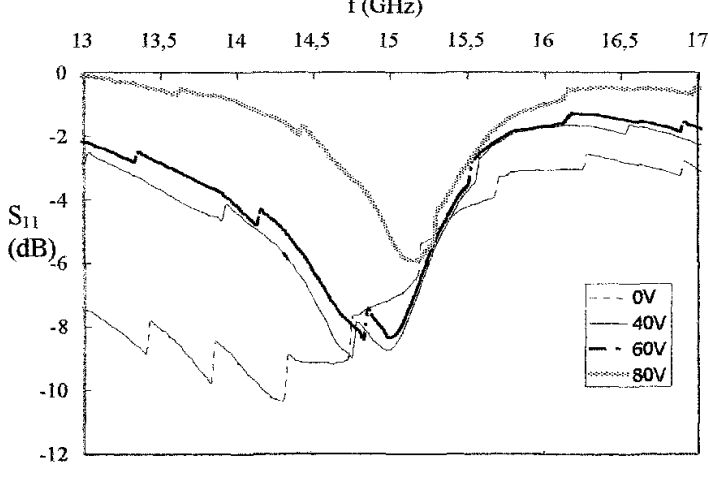

Figure 4: Paramètre $S_{11}(\mathrm{~dB})$ de la capacité pour différentes tensions appliquées à $77 \mathrm{~K}$

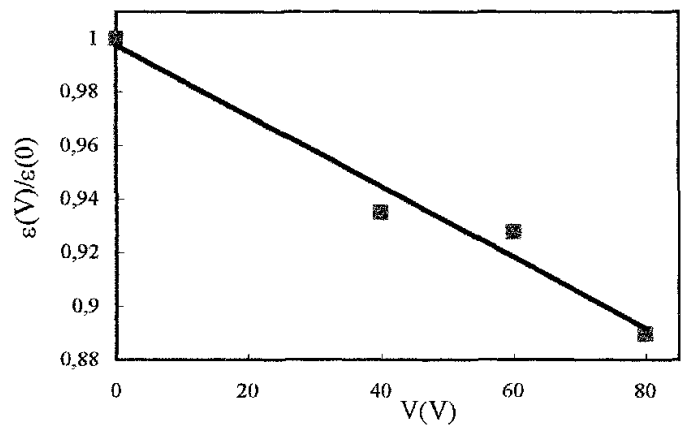

Figure 5: $\varepsilon_{\mathrm{T}}(\mathrm{V}) / \varepsilon_{\mathrm{r}}(\mathrm{OV})$ en fonction de la tension

Ces premiers résultats montrent qu'il est possible, sous l'action d'un champ électrique, de modifier la permittivité de nos films ferroélectriques. Cependant, pour améliorer les performances du composant, il est nécessaire d'adapter la composition du matériau pour un fonctionnement à $77 \mathrm{~K}$, c'est-à-dire diminuer le taux de baryum $x$. 


\section{NOUVEAUX COMPOSANTS : MEMS}

Depuis quelques années, les techniques classiques de fabrication de la microélectronique sont utilisées pour réaliser des objets Micro-Electro-Mécaniques (MEMS). Ces techniques permettent de réaliser des composants, des capteurs, voire des réacteurs intégrant des éléments mécaniques déformables (actionneurs). Aujourd'hui, ces micro-composants entrent dans les systèmes de télécommunications, où ils permettent d'apporter des solutions nouvelles et originales pour une nouvelle génération de composants accordables à faibles pertes et faible consommation [8]. Dans un premier temps nous nous focalisons, sur la conception et la réalisation de composants intégrant des capacités variables MEMs à base de ponts ou de poutres métalliques suspendus, utilisant des couches d'isolants, principalement alumine, élaborées par ablation laser. Diverses difficultés techniques doivent être maitrisées, qu'elles concernent la maitrise de la technologie microélectronique récemment mise en place, linsertion du procédé de dépôt par ablation laser dans la chaîne de fabrication, ou l'optimisation des paramètres expérimentaux, afin de satisfaire le cahier des charges du composant. Cependant, d'ores et déjà, plusieurs briques de base pour la réalisation de ces micro-objets sont validées (cf. figure 6).

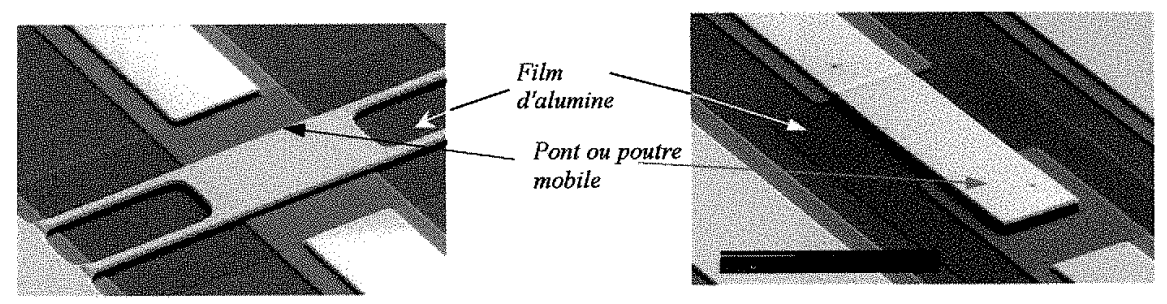

Figure 6: Capacités MEMs à base d'alumine réalisée par ablation laser.

A titre d'exemple, dans le cadre de ces activités, nous avons développé et fabriqué un micro commutateur RF [9] (cf figure 7). Ses pertes d'insertion de l'ordre de $-0,1 \mathrm{~dB}$, son isolation de l'ordre de $-40 \mathrm{~dB}$ le situent parmi les meilleurs publiés dans la littérature. Des études complémentaires sont en cours, elles concernent la corrélation entre les performances des commutateurs avec les propriétés microscopiques des matériaux diélectriques.

\section{CONCLUSION}

$\mathrm{S} 21(\mathrm{~dB})$

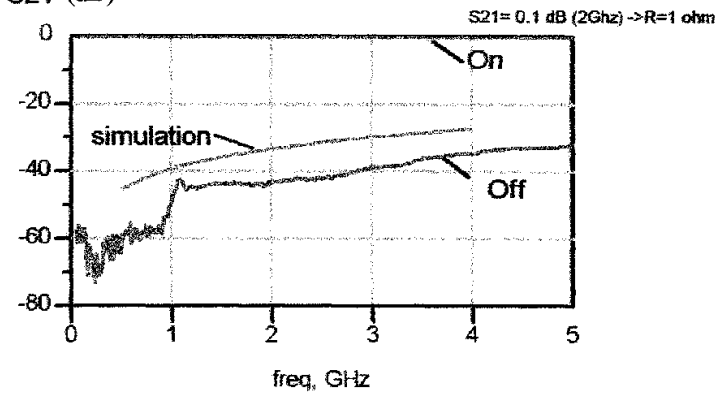

Figure 7 : Pertes d'insertion et isolation du commutateur.

Nos travaux concement l'application du procédé de dépôt par ablation laser à la réalisation de composants fonctionnant dans le domaine hyperfréquence. Des capacités variables sont réalisées, soit à partir d'hétérostructures de matériaux supraconducteur/ferroélectrique présentant une excellente hétéroépitaxie, soit à l'aide de structures MEMs. Ceci montre le potentiel du procédé d'ablation laser d'une part pour l'élaboration de nouveaux matériaux de bonne qualité cristalline et, d'autre part, par sa possible intégration dans une chaine de fabrication microélectronique.

\section{Références}

[1] J.S. Hong, M.J. Lancaster, D. Jedamzik, R.B. Greed, IEEE - MTT vol. 47 (9), Sept. 99.

[2] A. Tombak, F. Ayguavives, I. Maria, G. Stauf, A Kingon, A. Mortazawi, IEEE MTT-S Digest, 2001, 1453.

[3] G. Subramanyam, F. Van Keuls, F.A. Miranda. IEEE MTT-S Digest, 1998, 1011.

[4] T. Mitsui, Landolt-Bornstein, Springer Verlag, vol. 3, 1969.

[5] C. Champeaux, P. Marchet, A. Catherinot, App. Surf. Sci. 96-98, 775-778, 1996.

[6] T. Delage, C. Champeaux, A. Catherinot, J.F. Seaux, V. Madrangeas, D. Cros, J. Phys IV, ce numéro, 2002

[7] J.F. Seaux, D. Cros, V. Madrangeas, M. Aubourg, S. Verdeyme, Actes de JCMM, Toulouse, pp 207, 2002

[8] G.M. Rebeiz, J.B. Muldavin, IEEE Microwave Magazine, 59, 2001.

[9] A. Pothier, P. blondy, D. Cros, S. Verdeyme, P. Guillon, C. Champeaux, P. Tristant, A. Catherinot, accepté pour présentation EuMC Milan, sept. 2002. 